\title{
On New $p$-Valent Meromorphic Function Involving Certain Differential and Integral Operators
}

\author{
Aabed Mohammed ${ }^{1}$ and Maslina Darus ${ }^{2}$ \\ ${ }^{1}$ Basic Sciences Unit, Department of Mathematics, Sana'a Community College, Sana'a, Yemen \\ ${ }^{2}$ School of Mathematical Sciences, Faculty of Science and Technology, Universiti Kebangsaan Malaysia, 43600 Bangi, \\ Selangor D. Ehsan, Malaysia \\ Correspondence should be addressed to Maslina Darus; maslina@ukm.my
}

Received 21 August 2013; Revised 2 January 2014; Accepted 5 January 2014; Published 17 February 2014

Academic Editor: Marco Donatelli

Copyright (C) 2014 A. Mohammed and M. Darus. This is an open access article distributed under the Creative Commons Attribution License, which permits unrestricted use, distribution, and reproduction in any medium, provided the original work is properly cited.

We define new subclasses of meromorphic $p$-valent functions by using certain differential operator. Combining the differential operator and certain integral operator, we introduce a general $p$-valent meromorphic function. Then we prove the sufficient conditions for the function in order to be in the new subclasses.

\section{Introduction}

Let $\Sigma_{p}$ denote the class of meromorphic functions of the form

$$
f(z)=\frac{1}{z^{p}}+\sum_{n=p+1}^{\infty} a_{n} z^{n} \quad(p \in \mathbb{N}=\{1,2, \ldots\})
$$

which are analytic and $p$-valent in the punctured unit disc:

$$
\mathbb{U}^{*}=\{z \in \mathbb{C}: 0<|z|<1\}=\mathbb{U}-\{0\} .
$$

A function $f \in \Sigma_{p}$ is said to be in the class $\Sigma_{p}^{\star}(\delta)$ of meromorphic $p$-valent starlike of order $\delta(0 \leq \delta<p)$ if it satisfies the following inequality:

$$
-\Re\left(\frac{z f^{\prime}(z)}{f(z)}\right)>\delta .
$$

For $f \in \Sigma_{p}$, Saif and Kilıçman [1] introduced the linear operator $\mathscr{D}_{\lambda}^{k}$, as follows:

$$
\begin{gathered}
\mathscr{D}_{\lambda} f(z)=(1+p \lambda) f(z)+\lambda z f^{\prime}(z), \quad \lambda \geq 0, \\
\mathscr{D}_{\lambda}^{0} f(z)=f(z), \\
\mathscr{D}_{\lambda}^{1} f(z)=\mathscr{D}_{\lambda} f(z), \\
\mathscr{D}_{\lambda}^{2} f(z)=\mathscr{D}_{\lambda}\left(\mathscr{D}_{\lambda}^{1} f(z)\right),
\end{gathered}
$$

and in general, for $k=0,1,2, \ldots$, we can write

$$
\begin{array}{r}
\mathscr{D}_{\lambda}^{k} f(z)=\frac{1}{z^{p}}+\sum_{n=p+1}^{\infty}(1+p \lambda+n \lambda)^{k} a_{n} z^{n}, \\
\left(k \in \mathbb{N}_{0}=\mathbb{N} \cup\{0\}, p \in \mathbb{N}\right) .
\end{array}
$$

It is easy to see that, for $f \in \Sigma_{p}$, we have

$$
\begin{array}{r}
\lambda z\left(\mathscr{D}_{\lambda}^{k} f(z)\right)^{\prime}=\mathscr{D}_{\lambda}^{k+1} f(z)-(1+p \lambda) \mathscr{D}_{\lambda}^{k} f(z), \\
\left(k \in \mathbb{N}_{0}, p \in \mathbb{N}\right) .
\end{array}
$$

Meromorphically multivalent functions have been extensively studied by several authors; see, for example, Uralegaddi and Somanatha [2, 3], Liu and Srivastava [4, 5], Mogra [6, 7], Srivastava et al. [8], Aouf et al. [9, 10], Joshi and Srivastava [11], Owa et al. [12], and Kulkarni et al. [13].

Now, for $f \in \Sigma_{p}$, we define the following new subclasses.

Definition 1. Let a function $f \in \Sigma_{p}$ be analytic in $\mathbb{U}^{*}$. Then $f$ is in the class $\Sigma_{p} S_{k}(\delta, b, \lambda)$ if, and only if, $f$ satisfies

$$
\Re\left\{p-\frac{1}{b}\left(\frac{\mathscr{D}_{\lambda}^{k+1} f(z)}{\mathscr{D}_{\lambda}^{k} f(z)}-1\right)\right\}>\delta,
$$

where $\delta \in[0, p), b \in \mathbb{C} \backslash\{0\}, \lambda \geq 0, k \in \mathbb{N}_{0}$. 
From (6), one can see that (7) is equivalent to

$$
\mathfrak{R}\left\{p-\frac{\lambda}{b}\left(\frac{z\left(\mathscr{D}_{\lambda}^{k} f(z)\right)^{\prime}}{\mathscr{D}_{\lambda}^{k} f(z)}+p\right)\right\}>\delta .
$$

Remark 2. In Definition 1, if we set

(i) $k=0$ and $p=\lambda=1$, then we have [14, Definition 1.1];

(ii) $k=0$ and $p=\lambda=b=1$, then we have $\Sigma_{p}^{\star}(\delta)$, the class of meromorphic $p$-valent starlike of order $\delta$;

(iii) $k=1$ and $p=\lambda=1$, then we have [14, Definition 1.7].

Definition 3. Let a function $f \in \Sigma_{p}$ be analytic in $\mathbb{U}^{*}$. Then $f$ is in the class $\Sigma_{p} U S_{k}(\alpha, \delta, b, \lambda)$ if, and only if, $f$ satisfies

$$
\begin{aligned}
& \mathfrak{R}\left\{p-\frac{1}{b}\left(\frac{\mathscr{D}_{\lambda}^{k+1} f(z)}{\mathscr{D}_{\lambda}^{k} f(z)}-1\right)\right\} \\
& >\alpha\left|\frac{1}{b}\left(\frac{\mathscr{D}_{\lambda}^{k+1} f(z)}{\mathscr{D}_{\lambda}^{k} f(z)}-1\right)\right|+\delta,
\end{aligned}
$$

where $\alpha \geq 0, \delta \in[-1, p), b \in \mathbb{C} \backslash\{0\}, \lambda \geq 0, k \in \mathbb{N}_{0}$.

Inequality (9) is equivalent to

$$
\begin{aligned}
& \Re\left\{p-\frac{\lambda}{b}\left(\frac{z\left(\mathscr{D}_{\lambda}^{k} f(z)\right)^{\prime}}{\mathscr{D}_{\lambda}^{k} f(z)}+p\right)\right\} \\
& >\alpha\left|\frac{\lambda}{b}\left(\frac{z\left(\mathscr{D}_{\lambda}^{k} f(z)\right)^{\prime}}{\mathscr{D}_{\lambda}^{k} f(z)}+p\right)\right|+\delta .
\end{aligned}
$$

Remark 4. In Definition 3, if we set

(i) $k=0$ and $p=\lambda=1$, then we have [14, Definition 1.3];

(ii) for $k=1$ and $p=\lambda=1$, then we have [14, Definition $1.8]$.

Definition 5. Let a function $f \in \Sigma_{p}$ be analytic in $\mathbb{U}^{*}$. Then $f$ is in the class $\Sigma_{p} S H_{k}(\alpha, b, \lambda)$, if, and only if, $f$ satisfies

$$
\begin{aligned}
\mid p & -\frac{1}{b}\left(\frac{\mathscr{D}_{\lambda}^{k+1} f(z)}{\mathscr{D}_{\lambda}^{k} f(z)}-1\right)-2 \alpha(\sqrt{2}-1) \mid \\
& <\sqrt{2} \mathfrak{R}\left\{p-\frac{1}{b}\left(\frac{\mathscr{D}_{\lambda}^{k+1} f(z)}{\mathscr{D}_{\lambda}^{k} f(z)}-1\right)\right\}+2 \alpha(\sqrt{2}-1),
\end{aligned}
$$

where $\alpha>0, b \in \mathbb{C} \backslash\{0\}, \lambda \geq 0, k \in \mathbb{N}_{0}$.

Inequality (11) is equivalent to

$$
\begin{aligned}
\mid p & -\frac{\lambda}{b}\left(\frac{z\left(\mathscr{D}_{\lambda}^{k} f(z)\right)^{\prime}}{\mathscr{D}_{\lambda}^{k} f(z)}+p\right)-2 \alpha(\sqrt{2}-1) \mid \\
& <\sqrt{2} \mathfrak{R}\left\{p-\frac{\lambda}{b}\left(\frac{z\left(\mathscr{D}_{\lambda}^{k} f(z)\right)^{\prime}}{\mathscr{D}_{\lambda}^{k} f(z)}+p\right)\right\}+2 \alpha(\sqrt{2}-1) .
\end{aligned}
$$

Remark 6. In Definition 5, if we set

(i) $k=0$ and $p=\lambda=1$, then we have [14, Definition 1.5];

(ii) for $k=1$ and $p=\lambda=1$, then we have [14, Definition 1.9].

Recently, Mohammed and Darus [15] introduced the following $p$-valent meromorphic function:

$$
G(z)=z \mathscr{F}_{p, \gamma_{1}, \ldots, \gamma_{n}}^{\prime}(z)+(p+1) \mathscr{F}_{p, \gamma_{1}, \ldots, \gamma_{n}}(z),
$$

where $\mathscr{F}_{p, \gamma_{1}, \ldots, \gamma_{n}}$ is the integral operator introduced and studied by the authors $[15,16]$ and defined by

$$
\mathscr{F}_{p, \gamma_{1}, \ldots, \gamma_{n}}(z)=\frac{1}{z^{p+1}} \int_{0}^{z}\left(u^{p} f_{1}(u)\right)^{\gamma_{1}} \cdots\left(u^{p} f_{n}(u)\right)^{\gamma_{n}} d u,
$$

where

$$
n, p \in \mathbb{N}, \quad j \in\{1,2,3, \ldots, n\}, \quad \gamma_{j}>0 .
$$

For $p=1$ we obtain [17]. It is clear that

$$
G(z)=\frac{1}{z^{p}}\left(z^{p} f_{1}(z)\right)^{\gamma_{1}} \cdots\left(z^{p} f_{n}(z)\right)^{\gamma_{n}}
$$

By using the differential operator given by (4), we introduce the following $p$-valent meromorphic function.

Definition 7. Let $k \in \mathbb{N}_{0}, l=\left(l_{1}, \ldots, l_{n}\right) \in \mathbb{N}_{0}^{n}$ and $\gamma_{j}>$ $0,1 \leq j \leq n$. One defines the $p$-valent meromorphic function $I_{k, n, l, \gamma}: \sum_{p}^{n} \rightarrow \Sigma_{p}$,

$$
\begin{gathered}
I_{k, n, l, \gamma}\left(f_{1}, \ldots, f_{n}\right)=\Phi, \\
\mathscr{D}_{\lambda}^{k} \Phi(z)=\frac{1}{z^{p}}\left[\left(z^{p} \mathscr{D}_{\lambda}^{l_{1}} f_{1}(z)\right)^{\gamma_{1}} \cdots\left(z^{p} \mathscr{D}_{\lambda}^{l_{n}} f_{n}(z)\right)^{\gamma_{n}}\right],
\end{gathered}
$$

where $f_{1}, \ldots, f_{n} \in \Sigma_{p}$, and $\mathscr{D}_{\lambda}$ is the differential operator given by (4).

Remark 8. If we set $\lambda=1, k=0$, and $l_{1}=\cdots=l_{n}=0$, then we have the $p$-valent meromorphic function given by (13).

\section{Main Results}

To prove our main results, we need the following lemma.

Lemma 9. For the p-valent meromorphic function $I_{k, n, l, \gamma}\left(f_{1}\right.$, $\left.\ldots, f_{n}\right)=\Phi$ given by $(18)$, one has

$$
-\frac{\lambda z\left(\mathscr{D}_{\lambda}^{k} \Phi(z)\right)^{\prime}}{\mathscr{D}_{\lambda}^{k} \Phi(z)}=-\sum_{j=1}^{n} \gamma_{j} \frac{\mathscr{D}_{\lambda}^{l_{j}+1} f_{j}(z)}{\mathscr{D}_{\lambda}^{l_{j}} f_{j}(z)}+p \lambda+\sum_{j=1}^{n} \gamma_{j} .
$$

Proof. From (18), we have

$$
z^{p} \mathscr{D}_{\lambda}^{k} \Phi(z)=\left[\left(z^{p} \mathscr{D}_{\lambda}^{l_{1}} f_{1}(z)\right)^{\gamma_{1}} \cdots\left(z^{p} \mathscr{D}_{\lambda}^{l_{n}} f_{n}(z)\right)^{\gamma_{n}}\right] .
$$


Differentiating (20) logarithmically and then by simple computation, we get

$$
\frac{z\left(\mathscr{D}_{\lambda}^{k} \Phi(z)\right)^{\prime}}{\mathscr{D}_{\lambda}^{k} \Phi(z)}=\sum_{j=1}^{n} \gamma_{j}\left(\frac{z\left(\mathscr{D}_{\lambda}^{l_{j}} f_{j}(z)\right)^{\prime}+p \mathscr{D}_{\lambda}^{l_{j}} f_{j}(z)}{\mathscr{D}_{\lambda}^{l_{j}} f_{j}(z)}\right)-p .
$$

From (6), we obtain

$$
\left(D_{\lambda}^{l_{j}} f_{j}(z)\right)^{\prime}=\frac{\mathscr{D}_{\lambda}^{l_{j}+1} f_{j}(z)-(1+p \lambda) \mathscr{D}_{\lambda}^{l_{j}} f_{j}(z)}{\lambda z} .
$$

Then using (22) on the right-hand side of (21), one gets

$$
\frac{z\left(\mathscr{D}_{\lambda}^{k} \Phi(z)\right)^{\prime}}{\mathscr{D}_{\lambda}^{k} \Phi(z)}=\sum_{j=1}^{n} \gamma_{j}\left(\frac{\mathscr{D}_{\lambda}^{l_{j}+1} f_{j}(z)}{\lambda \mathscr{D}_{\lambda}^{l_{j}} f_{j}(z)}-\frac{1}{\lambda}\right)-p .
$$

Multiplying (23) by $\lambda$ yields that

$$
\frac{\lambda z\left(\mathscr{D}_{\lambda}^{k} \Phi(z)\right)^{\prime}}{\mathscr{D}_{\lambda}^{k} \Phi(z)}=\sum_{j=1}^{n} \gamma_{j}\left(\frac{\mathscr{D}_{\lambda}^{l_{j}+1} f_{j}(z)}{\mathscr{D}_{\lambda}^{l_{j}} f_{j}(z)}-1\right)-p \lambda,
$$

or, equivalently, we can write that

$$
-\frac{\lambda z\left(\mathscr{D}_{\lambda}^{k} \Phi(z)\right)^{\prime}}{\mathscr{D}_{\lambda}^{k} \Phi(z)}=-\sum_{j=1}^{n} \gamma_{j} \frac{\mathscr{D}_{\lambda}^{l_{j}+1} f_{j}(z)}{\mathscr{D}_{\lambda}^{l_{j}} f_{i}(z)}+p \lambda+\sum_{j=1}^{n} \gamma_{j},
$$

which is the desired result.

Our first theorem is as follows.

Theorem 10. Let $\alpha_{j} \geq 0, \delta_{j} \in[-1, p), \alpha_{j}+\delta_{j} \geq 0, \quad(1 \leq j \leq$ $n)$ and $b \in \mathbb{C} \backslash\{0\}, \lambda \geq 0$. Suppose that

$$
\sum_{j=1}^{n} \gamma_{j}\left(\frac{p-\delta_{j}}{\alpha_{j}+1}\right) \leq p
$$

If $f_{j} \in \Sigma_{p} U S_{l_{j}}\left(\alpha_{j}, \delta_{j}, b, \lambda\right)(1 \leq j \leq n)$, then the function $\mathscr{D}_{\lambda}^{k} \Phi(z)$ defined by (18) is in the class $\Sigma_{p} S_{k}(\mu, b, \lambda)$, where

$$
\mu=p-\sum_{j=1}^{n} \gamma_{j}\left(\frac{p-\delta_{j}}{\alpha_{j}+1}\right) .
$$

Proof. Since $f_{j} \in \Sigma_{p} U S_{l_{j}}\left(\alpha_{j}, \delta_{j}, b, \lambda\right)(1 \leq j \leq n)$, by (9), we have

$$
\Re\left\{p-\frac{1}{b}\left(\frac{\mathscr{D}_{\lambda}^{l_{j}+1} f_{j}(z)}{\mathscr{D}_{\lambda}^{l_{j}} f_{j}(z)}-1\right)\right\}>\frac{p \alpha_{j}+\delta_{j}}{1+\alpha_{j}} .
$$

By (19), we get

$$
-\frac{\lambda z\left(\mathscr{D}_{\lambda}^{k} \Phi(z)\right)^{\prime}}{\mathscr{D}_{\lambda}^{k} \Phi(z)}-p \lambda=-\sum_{j=1}^{n} \gamma_{j}\left(\frac{\mathscr{D}_{\lambda}^{l_{j}+1} f_{j}(z)}{\mathscr{D}_{\lambda}^{l_{j}} f_{j}(z)}-1\right) .
$$

This is equivalent to

$$
\begin{aligned}
p & -\frac{\lambda}{b}\left(\frac{z\left(\mathscr{D}_{\lambda}^{k} \Phi(z)\right)^{\prime}}{\mathscr{D}_{\lambda}^{k} \Phi(z)}+p\right) \\
& =p-\frac{1}{b} \sum_{j=1}^{n} \gamma_{j}\left(\frac{\mathscr{D}_{\lambda}^{l_{j}+1} f_{j}(z)}{\mathscr{D}_{\lambda}^{l_{j}} f_{j}(z)}-1\right) \\
& =\sum_{j=1}^{n} \gamma_{j}\left[p-\frac{1}{b}\left(\frac{\mathscr{D}_{\lambda}^{l_{j}+1} f_{j}(z)}{\mathscr{D}_{\lambda}^{l_{j}} f_{j}(z)}-1\right)\right]+p-p \sum_{j=1}^{n} \gamma_{j} .
\end{aligned}
$$

From (28) together with (30), we can get

$$
\begin{aligned}
\Re & \left\{p-\frac{\lambda}{b}\left(\frac{z\left(\mathscr{D}_{\lambda}^{k} \Phi(z)\right)^{\prime}}{\mathscr{D}_{\lambda}^{k} \Phi(z)}+p\right)\right\} \\
= & \sum_{j=1}^{n} \gamma_{j} \mathfrak{R}\left[p-\frac{1}{b}\left(\frac{\mathscr{D}_{\lambda}^{l_{j}+1} f_{j}(z)}{\mathscr{D}_{\lambda}^{l_{j}} f_{j}(z)}-1\right)\right] \\
& +p-p \sum_{j=1}^{n} \gamma_{j} \\
> & \sum_{j=1}^{n} \gamma_{j}\left(\frac{p \alpha_{j}+\delta_{j}}{1+\alpha_{j}}\right)-p \sum_{j=1}^{n} \gamma_{j}+p \\
= & p-\sum_{j=1}^{n} \gamma_{j}\left(\frac{p-\delta_{j}}{1+\alpha_{j}}\right) .
\end{aligned}
$$

Hence, we obtain $\mathscr{D}_{\lambda}^{k} \Phi(z) \in \Sigma_{p} S_{k}(\mu, b, \lambda)$, where $\mu=p-$ $\sum_{j=1}^{n} \gamma_{j}\left(\left(p-\delta_{j}\right) /\left(\alpha_{j}+1\right)\right)$.

Corollary 11. Let $\alpha_{j} \geq 0, \delta_{j} \in[-1, p), \alpha_{j}+\delta_{j} \geq 0, \quad(1 \leq j \leq$ $n)$, and $b \in \mathbb{C} \backslash\{0\}, \lambda \geq 0$. Suppose that

$$
\sum_{j=1}^{n} \gamma_{j}\left(\frac{p-\delta_{j}}{\alpha_{j}+1}\right) \leq p .
$$

If $f_{j} \in \Sigma_{p} U S_{l_{j}}\left(\alpha_{j}, \delta_{j}, b, 1\right)(1 \leq j \leq n)$, then the function $\mathscr{D}_{\lambda}^{k} \Phi(z)$, defined by (18), is in the class $\Sigma_{p} S_{k+1}(\mu, b, 1)$, where $\mu$ is defined as in (27).

Proof. In Theorem 10, we consider $\lambda=1$.

By Corollary 11, we easily get the following.

Corollary 12. Let $\alpha_{j} \geq 0, \delta_{j} \in[-1, p), \alpha_{j}+\delta_{j} \geq 0,(1 \leq j \leq$ $n)$, and $b \in \mathbb{C} \backslash\{0\}, \lambda \geq 0$. Suppose that

$$
\sum_{j=1}^{n} \gamma_{j}\left(\frac{p-\delta_{j}}{\alpha_{j}+1}\right) \leq p .
$$

If $f_{j} \in \Sigma_{p} U S_{l_{j}}\left(\alpha_{j}, \delta_{j}, b, 1\right)(1 \leq j \leq n)$, then the function $\mathscr{D}_{\lambda}^{k} \Phi(z)$, defined by (18), is in the class $\Sigma_{p} S_{k+1}(0, b, 1)$. 
Now, we prove a sufficient condition for the function $\mathscr{D}_{\lambda}^{k}$ $\Phi(z)$ defined by (18) to belong to the class $\Sigma_{p} U S_{k}(\alpha, \delta, b, \lambda)$.

Theorem 13. Let $\alpha \geq 0, \delta \in[-1, p), \alpha+\delta \geq 0 \quad(1 \leq j \leq n)$, and $b \in \mathbb{C} \backslash\{0\}, \lambda \geq 0$. Suppose that

$$
\sum_{j=1}^{n} \gamma_{j} \leq 1
$$

If $f_{j} \in \Sigma_{p} U S_{l_{j}}(\alpha, \delta, b, \lambda)(1 \leq j \leq n)$, then the function $\mathscr{D}_{\lambda}^{k}$ $\Phi(z)$ defined by (18) is in the class $\Sigma_{p} U S_{k}(\alpha, \delta, b, \lambda)$.

Proof. Since $f_{j} \in \Sigma_{p} U S_{l_{j}}(\alpha, \delta, b, \lambda)(1 \leq j \leq n)$, by (9), we have

$$
\begin{aligned}
& \Re\left\{p-\frac{1}{b}\left(\frac{\mathscr{D}_{\lambda}^{l_{j}+1} f(z)}{\mathscr{D}_{\lambda}^{l_{j}} f(z)}-1\right)\right\} \\
& >\alpha\left|\frac{1}{b}\left(\frac{\mathscr{D}_{\lambda}^{l_{j}+1} f_{j}(z)}{\mathscr{D}_{\lambda}^{l_{j}} f_{j}(z)}-1\right)\right|+\delta .
\end{aligned}
$$

On the other hand, from (19), we obtain the following:

$$
\begin{aligned}
p & -\frac{\lambda}{b}\left(\frac{z\left(\mathscr{D}_{\lambda}^{k} \Phi(z)\right)^{\prime}}{\mathscr{D}_{\lambda}^{k} \Phi(z)}+p\right) \\
& =\sum_{j=1}^{n} \gamma_{j}\left[p-\frac{1}{b}\left(\frac{\mathscr{D}_{\lambda}^{l_{j}+1} f_{j}(z)}{\mathscr{D}_{\lambda}^{l_{j}} f_{j}(z)}-1\right)\right]+p-p \sum_{j=1}^{n} \gamma_{j} .
\end{aligned}
$$

Considering (10) with the above equality, we find

$$
\begin{aligned}
& \mathfrak{R}\left\{p-\frac{\lambda}{b}\left(\frac{z\left(\mathscr{D}_{\lambda}^{k} f(z)\right)^{\prime}}{\mathscr{D}_{\lambda}^{k} f(z)}+p\right)\right\} \\
& \quad-\alpha\left|\frac{\lambda}{b}\left(\frac{z\left(\mathscr{D}_{\lambda}^{k} f(z)\right)^{\prime}}{\mathscr{D}_{\lambda}^{k} f(z)}+p\right)\right|-\delta \\
& =p-p \sum_{j=1}^{n} \gamma_{j}+\sum_{j=1}^{n} \gamma_{j} \mathfrak{R}\left[p-\frac{1}{b}\left(\frac{\mathscr{D}_{\lambda}^{l_{j}+1} f_{j}(z)}{\mathscr{D}_{\lambda}^{l_{j}} f_{j}(z)}-1\right)\right]
\end{aligned}
$$

$$
\begin{aligned}
& -\alpha\left|\sum_{j=1}^{n} \gamma_{j} \frac{1}{b}\left(\frac{\mathscr{D}_{\lambda}^{l_{j}+1} f_{j}(z)}{\mathscr{D}_{\lambda}^{l_{j}} f_{j}(z)}-1\right)\right|-\delta \\
\geq & p-p \sum_{j=1}^{n} \gamma_{j}+\sum_{j=1}^{n} \gamma_{j} \mathfrak{R}\left[p-\frac{1}{b}\left(\frac{\mathscr{D}_{\lambda}^{l_{j}+1} f_{j}(z)}{\mathscr{D}_{\lambda}^{l_{j}} f_{j}(z)}-1\right)\right] \\
& -\alpha \sum_{j=1}^{n} \gamma_{j}\left|\frac{1}{b}\left(\frac{\mathscr{D}_{\lambda}^{l_{j}+1} f_{j}(z)}{\mathscr{D}_{\lambda}^{l_{j}} f_{j}(z)}-1\right)\right|-\delta \\
> & p-p \sum_{j=1}^{n} \gamma_{j}+\sum_{j=1}^{n} \gamma_{j}\left[\alpha\left|\frac{1}{b}\left(\frac{\mathscr{D}_{\lambda}^{l_{j}+1} f_{j}(z)}{\mathscr{D}_{\lambda}^{l_{j}} f_{j}(z)}-1\right)\right|+\delta\right] \\
& -\alpha \sum_{j=1}^{n} \gamma_{j}\left|\frac{1}{b}\left(\frac{\mathscr{D}_{\lambda}^{l_{j}+1} f_{j}(z)}{\mathscr{D}_{\lambda}^{l_{j}} f_{j}(z)}-1\right)\right|-\delta \\
= & (p-\delta)\left(1-\sum_{j=1}^{n} \gamma_{j}\right) \geq 0 .
\end{aligned}
$$

The proof is complete.

Corollary 14. Let $\alpha \geq 0, \delta \in[-1, p), \alpha+\delta \geq 0(1 \leq j \leq n)$, and $b \in \mathbb{C} \backslash\{0\}$. Suppose that

$$
\sum_{j=1}^{n} \gamma_{j} \leq 1
$$

If $f_{j} \in \Sigma_{p} U S_{l_{j}}(\alpha, \delta, b, 1)(1 \leq j \leq n)$, then the function $\mathscr{D}_{\lambda}^{k} \Phi(z)$ defined by (18) is in the class $\Sigma_{p} U S_{k+1}(\alpha, \delta, b, 1)$.

Proof. In Theorem 13, we consider that $\lambda=1$

Next, for the function $\mathscr{D}_{\lambda}^{k} \Phi$ defined by (18) to belong to the class $\Sigma_{p} S H_{k}(\alpha, b, \lambda)$, we have the following result.

Theorem 15. Let $\alpha \geq 0, \lambda \geq 0$, and $b \in \mathbb{C} \backslash\{0\}$. Suppose that

$$
\sum_{j=1}^{n} \gamma_{j} \leq 1
$$

If $f_{j} \in \Sigma_{p} S H_{l_{j}}(\alpha, b, \lambda)$, then the function $\mathscr{D}_{\lambda}^{k} \Phi(z) \in \Sigma_{p} S H_{k}(\alpha$, $b, \lambda)$.

Proof. Since $f_{j} \in \Sigma_{p} S H_{l_{j}}(\alpha, b, \lambda)$, by (11), we have

$$
\begin{aligned}
\sqrt{2} \mathfrak{R} & \left\{p-\frac{1}{b}\left(\frac{\mathscr{D}_{\lambda}^{l_{j}+1} f_{j}(z)}{\mathscr{D}_{\lambda}^{l_{j}} f_{j}(z)}-1\right)\right\}+2 \alpha(\sqrt{2}-1) \\
& -\left|p-\frac{1}{b}\left(\frac{\mathscr{D}_{\lambda}^{l_{j}+1} f_{j}(z)}{\mathscr{D}_{\lambda}^{l_{j}} f_{j}(z)}-1\right)-2 \alpha(\sqrt{2}-1)\right|>0 .
\end{aligned}
$$


Combining (12), (30), and the above inequality, we obtain

$$
\begin{aligned}
-\mid \sum_{j=1}^{n} \gamma_{j}\left\{\left(p-\frac{1}{b}\left(\frac{\mathscr{D}_{\lambda}^{l_{j}+1} f_{j}(z)}{\mathscr{D}_{\lambda}^{l_{j}} f_{j}(z)}-1\right)\right)\right. \\
-2 \alpha(\sqrt{2}-1)\} \\
+[p-2 \alpha(\sqrt{2}-1)]\left(1-\sum_{j=1}^{n} \gamma_{j}\right) \mid,
\end{aligned}
$$$$
\sqrt{2} \mathfrak{R}\left\{p-\frac{\lambda}{b}\left(\frac{z\left(\mathscr{D}_{\lambda}^{k} f(z)\right)^{\prime}}{\mathscr{D}_{\lambda}^{k} f(z)}+p\right)\right\}+2 \alpha(\sqrt{2}-1)
$$$$
-\left|p-\frac{\lambda}{b}\left(\frac{z\left(\mathscr{D}_{\lambda}^{k} f(z)\right)^{\prime}}{\mathscr{D}_{\lambda}^{k} f(z)}+p\right)-2 \alpha(\sqrt{2}-1)\right|
$$$$
=\sqrt{2} \Re\left\{\sum_{j=1}^{n} \gamma_{j}\left[p-\frac{1}{b}\left(\frac{\mathscr{D}_{\lambda}^{l_{j}+1} f_{j}(z)}{\mathscr{D}_{\lambda}^{l_{j}} f_{j}(z)}-1\right)\right]\right.
$$$$
\left.+p-p \sum_{j=1}^{n} \gamma_{j}\right\}+2 \alpha(\sqrt{2}-1)
$$$$
-\mid \sum_{j=1}^{n} \gamma_{j}\left[p-\frac{1}{b}\left(\frac{\mathscr{D}_{\lambda}^{l_{j}+1} f_{j}(z)}{\mathscr{D}_{\lambda}^{l_{j}} f_{j}(z)}-1\right)\right]
$$$$
+p-p \sum_{j=1}^{n} \gamma_{j}-2 \alpha(\sqrt{2}-1)
$$$$
=\sum_{j=1}^{n} \gamma_{j}\left\{\sqrt{2} \mathfrak{R}\left(p-\frac{1}{b}\left(\frac{\mathscr{D}_{\lambda}^{l_{j}+1} f_{j}(z)}{\mathscr{D}_{\lambda}^{l_{j}} f_{j}(z)}-1\right)\right)\right.
$$$$
+2 \alpha(\sqrt{2}-1)\}-2 \alpha(\sqrt{2}-1) \sum_{j=1}^{n} \gamma_{j}
$$$$
+\sqrt{2}\left(p-p \sum_{j=1}^{n} \gamma_{j}\right)+2 \alpha(\sqrt{2}-1)
$$$$
-\mid \sum_{j=1}^{n} \gamma_{j}\left\{\left(p-\frac{1}{b}\left(\frac{\mathscr{D}_{\lambda}^{l_{j}+1} f_{j}(z)}{\mathscr{D}_{\lambda}^{l_{j}} f_{j}(z)}-1\right)\right)\right.
$$$$
-2 \alpha(\sqrt{2}-1)\}
$$$$
+2 \alpha(\sqrt{2}-1) \sum_{j=1}^{n} \gamma_{j}-2 \alpha(\sqrt{2}-1)+p-p \sum_{j=1}^{n} \gamma_{j}
$$

which is

$$
\begin{aligned}
& \geq \sum_{j=1}^{n} \gamma_{j}\left\{\sqrt{2} \mathfrak{R}\left(p-\frac{1}{b}\left(\frac{\mathscr{D}_{\lambda}^{l_{j}+1} f_{j}(z)}{\mathscr{D}_{\lambda}^{l_{j}} f_{j}(z)}-1\right)\right)\right. \\
& +2 \alpha(\sqrt{2}-1)\} \\
& +[\sqrt{2} p+2 \alpha(\sqrt{2}-1)]\left(1-\sum_{j=1}^{n} \gamma_{j}\right) \\
& -\sum_{j=1}^{n} \gamma_{j} \mid\left\{\left(p-\frac{1}{b}\left(\frac{\mathscr{D}_{\lambda}^{l_{j}+1} f_{j}(z)}{\mathscr{D}_{\lambda}^{l_{j}} f_{j}(z)}-1\right)\right)\right. \\
& -2 \alpha(\sqrt{2}-1)\} \\
& -|p-2 \alpha(\sqrt{2}-1)|\left(1-\sum_{j=1}^{n} \gamma_{j}\right) \\
& =\sum_{j=1}^{n} \gamma_{j}\left\{\sqrt{2} \mathfrak{R}\left[p-\frac{1}{b}\left(\frac{\mathscr{D}_{\lambda}^{l_{j}+1} f_{j}(z)}{\mathscr{D}_{\lambda}^{l_{j}} f_{j}(z)}-1\right)\right]\right. \\
& +2 \alpha(\sqrt{2}-1) \\
& \left.-\left|p-\frac{1}{b}\left(\frac{\mathscr{D}_{\lambda}^{l_{j}+1} f_{j}(z)}{\mathscr{D}_{\lambda}^{l_{j}} f_{j}(z)}-1\right)-2 \alpha(\sqrt{2}-1)\right|\right\} \\
& +[\sqrt{2} p+2 \alpha(\sqrt{2}-1)-|p-2 \alpha(\sqrt{2}-1)|] \\
& \times\left(1-\sum_{j=1}^{n} \gamma_{j}\right) \\
& >[\sqrt{2} p+2 \alpha(\sqrt{2}-1)-|p-2 \alpha(\sqrt{2}-1)|] \\
& \times\left(1-\sum_{j=1}^{n} \gamma_{j}\right)
\end{aligned}
$$

$$
\begin{aligned}
=\sum_{j=1}^{n} \gamma_{j}\{ & \sqrt{2} \mathfrak{R}\left(p-\frac{1}{b}\left(\frac{\mathscr{D}_{\lambda}^{l_{j}+1} f_{j}(z)}{\mathscr{D}_{\lambda}^{l_{j}} f_{j}(z)}-1\right)\right) \\
& +2 \alpha(\sqrt{2}-1)\} \\
& +[\sqrt{2} p+2 \alpha(\sqrt{2}-1)]\left(1-\sum_{j=1}^{n} \gamma_{j}\right)
\end{aligned}
$$


and finally

$$
>\left(1-\sum_{j=1}^{n} \gamma_{j}\right) \min \{(\sqrt{2}-1)(p+4 \alpha), p(\sqrt{2}+1)\} \geq 0
$$

Hence, by (12), we have $\mathscr{D}_{\lambda}^{k} \Phi(z) \in \Sigma_{p} S H_{k}(\alpha, b, \lambda)$.

Corollary 16. Let $\alpha \geq 0$ and $b \in \mathbb{C} \backslash\{0\}$. Suppose that

$$
\sum_{j=1}^{n} \gamma_{j} \leq 1
$$

If $f_{j} \in \Sigma_{p} S H_{l_{j}}(\alpha, b, 1)$, then the function $\mathscr{D}_{\lambda}^{k} \Phi(z)$ defined by (18) is in the class $\Sigma_{p} S H_{k+1}(\alpha, b, 1)$.

Proof. In Theorem 15, we consider $\lambda=1$.

Finally, we end this paper by the following theorem and its consequence.

Theorem 17. Let $\alpha \geq 0, \lambda \geq 0$, and $b \in \mathbb{C} \backslash\{0\}$. Suppose that

$$
(p+\sqrt{2} \alpha(\sqrt{2}-1)) \sum_{j=1}^{n} \gamma_{j}<p
$$

If $f_{j} \in \Sigma_{p} S H_{l_{j}}(\alpha, b, \lambda)$, then the function $\mathscr{D}_{\lambda}^{k} \Phi(z)$ defined by (18) is in the class $\Sigma_{p} S H_{k}(0, b, \lambda)$.

Proof. Since $f_{j} \in \Sigma_{p} S H_{l_{j}}(\alpha, b, \lambda)$, by (11), we have

$$
\begin{gathered}
\sqrt{2} \mathfrak{R}\left\{p-\frac{1}{b}\left(\frac{\mathscr{D}_{\lambda}^{l_{j}+1} f_{j}(z)}{\mathscr{D}_{\lambda}^{l_{j}} f_{j}(z)}-1\right)\right\}+2 \alpha(\sqrt{2}-1) \\
>\left|p-\frac{1}{b}\left(\frac{\mathscr{D}_{\lambda}^{l_{j}+1} f_{j}(z)}{\mathscr{D}_{\lambda}^{l_{j}} f_{j}(z)}-1\right)-2 \alpha(\sqrt{2}-1)\right| .
\end{gathered}
$$

Considering this inequality and (30), we obtain

$$
\begin{aligned}
\sqrt{2} \mathfrak{R}\left\{p-\frac{\lambda}{b}\left(\frac{z\left(\mathscr{D}_{\lambda}^{k} f(z)\right)^{\prime}}{\mathscr{D}_{\lambda}^{k} f(z)}+p\right)\right\} \\
=\sqrt{2} \mathfrak{R}\left\{\sum_{j=1}^{n} \gamma_{j}\left[p-\frac{1}{b}\left(\frac{\mathscr{D}_{\lambda}^{l_{j}+1} f_{j}(z)}{\mathscr{D}_{\lambda}^{l_{j}} f_{j}(z)}-1\right)\right]\right.
\end{aligned}
$$

$$
\begin{gathered}
\left.+p-p \sum_{j=1}^{n} \gamma_{j}\right\} \\
=\sum_{j=1}^{n} \gamma_{j}\left\{\sqrt{2} \mathfrak{R}\left[p-\frac{1}{b}\left(\frac{\mathscr{D}_{\lambda}^{l_{j}+1} f_{j}(z)}{\mathscr{D}_{\lambda}^{l_{j}} f_{j}(z)}-1\right)\right]\right. \\
+2 \alpha(\sqrt{2}-1)\}
\end{gathered}
$$

$$
\begin{aligned}
& +\sqrt{2} p\left(1-\sum_{j=1}^{n} \gamma_{j}\right)-2 \alpha(\sqrt{2}-1) \sum_{j=1}^{n} \gamma_{j} \\
> & \sqrt{2} p\left(1-\sum_{j=1}^{n} \gamma_{j}\right)-2 \alpha(\sqrt{2}-1) \sum_{j=1}^{n} \gamma_{j} \\
= & \sqrt{2}\left(p-(p+\sqrt{2} \alpha(\sqrt{2}-1)) \sum_{j=1}^{n} \gamma_{j}\right)>0 .
\end{aligned}
$$

Hence, we have $\mathscr{D}_{\lambda}^{k} \Phi(z) \in \Sigma_{p} S H_{k}(0, b, \lambda)$.

Corollary 18. Let $\alpha \geq 0$ and $b \in \mathbb{C} \backslash\{0\}$. Suppose that

$$
(p+\sqrt{2} \alpha(\sqrt{2}-1)) \sum_{j=1}^{n} \gamma_{j}<p
$$

If $f_{j} \in \Sigma_{p} S H_{l_{j}}(\alpha, b, 1)$, then the function $\mathscr{D}_{\lambda}^{k} \Phi(z)$ defined by (18) is in the class $\Sigma_{p} S H_{k+1}(0, b, 1)$.

Proof. In Theorem 17, we consider that $\lambda=1$.

For other work that we can look at regarding differential and integral operators, see [14, 18-24].

\section{Conflict of Interests}

The authors declare that there is no conflict of interests regarding the publication of this paper.

\section{Acknowledgment}

The work here is fully supported by UKM's Grants: AP-2013009 and DIP-2013-001.

\section{References}

[1] A. Saif and A. Kılıçman, "On certain subclasses of meromorphically $p$-valent functions associated by the linear operator $D_{\lambda}^{n}$," Journal of Inequalities and Applications, vol. 2011, Article ID 401913, 16 pages, 2011.

[2] B. A. Uralegaddi and C. Somanatha, "New criteria for meromorphic starlike univalent functions," Bulletin of the Australian Mathematical Society, vol. 43, no. 1, pp. 137-140, 1991.

[3] B. A. Uralegaddi and C. Somanatha, "Certain classes of meromorphic multivalent functions," Tamkang Journal of Mathematics, vol. 23, no. 3, pp. 223-231, 1992. 
[4] J.-L. Liu and H. M. Srivastava, "A linear operator and associated families of meromorphically multivalent functions," Journal of Mathematical Analysis and Applications, vol. 259, no. 2, pp. 566$581,2001$.

[5] J.-L. Liu and H. M. Srivastava, "Some convolution conditions for starlikeness and convexity of meromorphically multivalent functions," Applied Mathematics Letters, vol. 16, no. 1, pp. 13-16, 2003.

[6] M. L. Mogra, "Meromorphic multivalent functions with positive coefficients. I," Mathematica Japonica, vol. 35, no. 1, pp. 1-11, 1990.

[7] M. L. Mogra, "Meromorphic multivalent functions with positive coefficients. II," Mathematica Japonica, vol. 35, no. 6, pp. 1089-1098, 1990.

[8] H. M. Srivastava, H. M. Hossen, and M. K. Aouf, "A unified presentation of some classes of meromorphically multivalent functions," Computers \& Mathematics with Applications, vol. 38, no. 11-12, pp. 63-70, 1999.

[9] M. K. Aouf and H. M. Hossen, "New criteria for meromorphic p-valent starlike functions," Tsukuba Journal of Mathematics, vol. 17, no. 2, pp. 481-486, 1993.

[10] M. K. Aouf and H. M. Srivastava, "A new criterion for meromorphically $p$-valent convex functions of order alpha," Mathematical Sciences Research Hot-Line, vol. 1, no. 8, pp. 7-12, 1997.

[11] S. B. Joshi and H. M. Srivastava, "A certain family of meromorphically multivalent functions," Computers \& Mathematics with Applications, vol. 38, no. 3-4, pp. 201-211, 1999.

[12] S. Owa, H. E. Darwish, and M. K. Aouf, "Meromorphic multivalent functions with positive and fixed second coefficients," Mathematica Japonica, vol. 46, no. 2, pp. 231-236, 1997.

[13] S. R. Kulkarni, U. H. Naik, and H. M. Srivastava, "A certain class of meromorphically p-valent quasi-convex functions," Panamerican Mathematical Journal, vol. 8, no. 1, pp. 57-64, 1998.

[14] A. Mohammed and M. Darus, "Integral operators on new families of meromorphic functions of complex order," Journal of Inequalities and Applications, vol. 2011, article 121, 12 pages, 2011.

[15] A. Mohammed and M. Darus, "The order of starlikeness of new $p$-valent meromorphic functions," International Journal of Mathematical Analysis, vol. 6, no. 25-28, pp. 1329-1340, 2012.

[16] A. Mohammed and M. Darus, "Some properties of certain integral operators on new subclasses of analytic functions with complex order," Journal of Applied Mathematics, vol. 2012, Article ID 161436, 9 pages, 2012.

[17] A. Mohammed and M. Darus, "A new integral operator for meromorphic functions," Acta Universitatis Apulensis, no. 24, pp. 231-238, 2010.

[18] A. Mohammed and M. Darus, "New properties for certain integral operators," International Journal of Mathematical Analysis, vol. 4, no. 42, pp. 2101-2109, 2010.

[19] A. Mohammed and M. Darus, "Starlikeness properties of a new integral operator for meromorphic functions," Journal of Applied Mathematics, vol. 2011, Article ID 804150, 8 pages, 2011.

[20] B. A. Frasin, "On an integral operator of meromorphic functions," Matematichki Vesnik, vol. 64, no. 2, pp. 167-172, 2012.

[21] N. Breaz, D. Breaz, and M. Darus, "Convexity properties for some general integral operators on uniformly analytic functions classes," Computers \& Mathematics with Applications, vol. 60, no. 12, pp. 3105-3107, 2010.
[22] S. Bulut, "Some properties for an integral operator defined by Al-Oboudi differential operator," Journal of Inequalities in Pure and Applied Mathematics, vol. 9, no. 4, article 115, 5 pages, 2008.

[23] S. Bulut, "A new general integral operator defined by Al-Oboudi differential operator," Journal of Inequalities and Applications, vol. 2009, Article ID 158408, 13 pages, 2009.

[24] P. Goswami and S. Bulut, "Starlikeness of general integral operator for meromorphic multivalent functions," Journal of Complex Analysis, vol. 2013, Article ID 690584, 4 pages, 2013. 


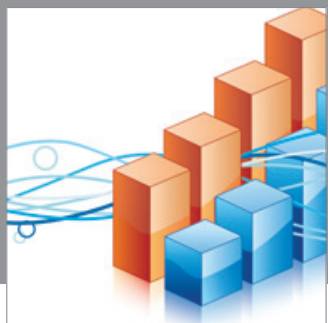

Advances in

Operations Research

mansans

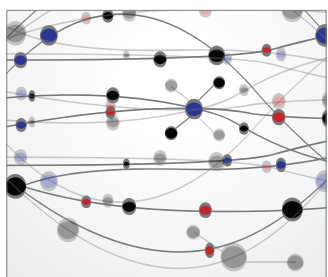

The Scientific World Journal
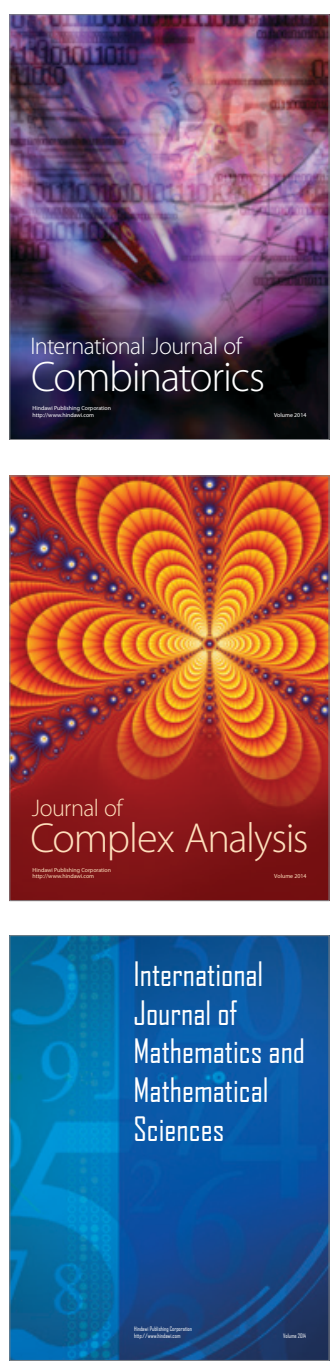
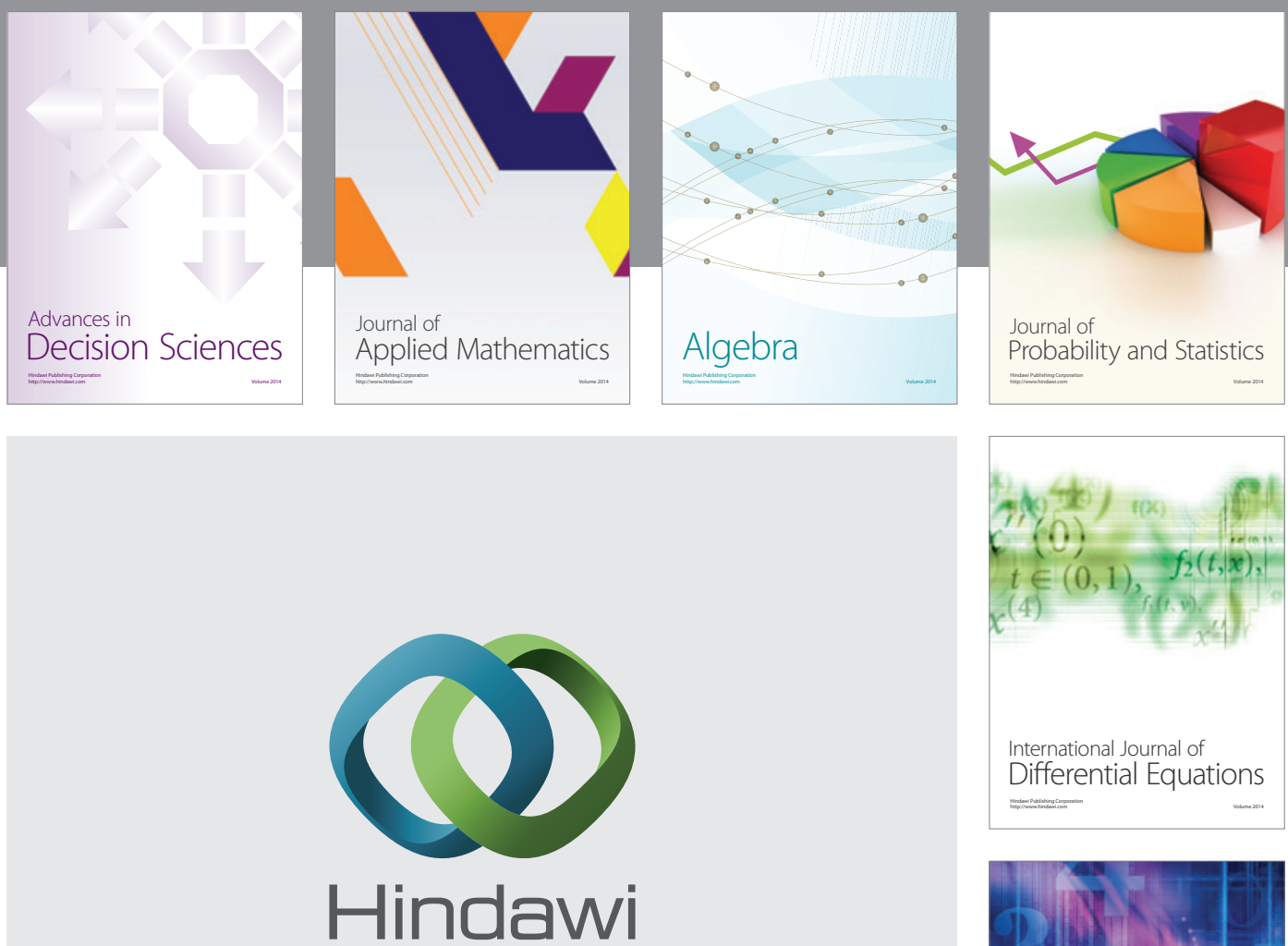

Submit your manuscripts at http://www.hindawi.com
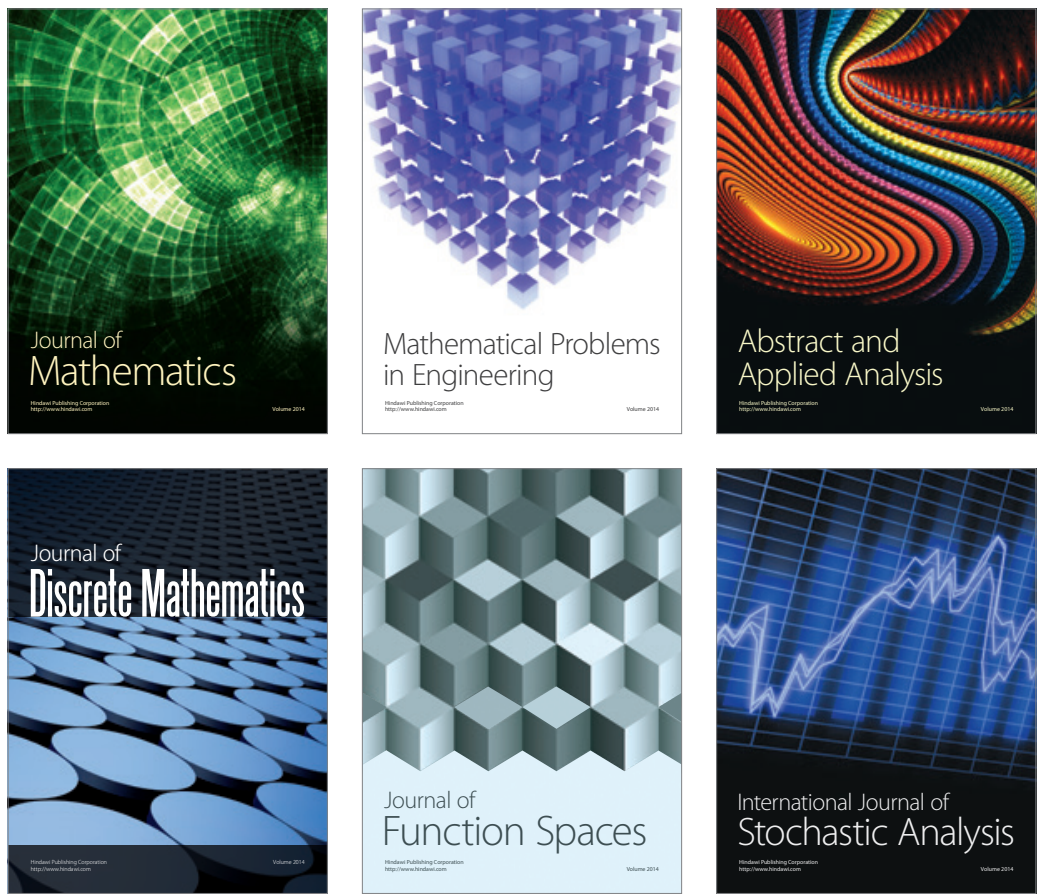

Journal of

Function Spaces

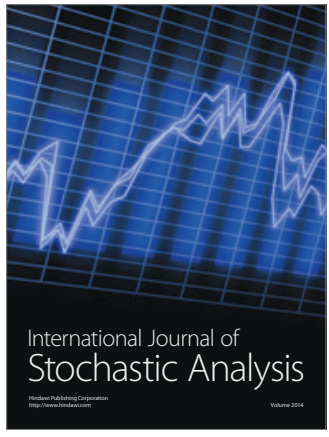

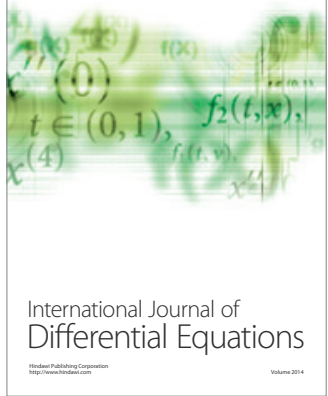
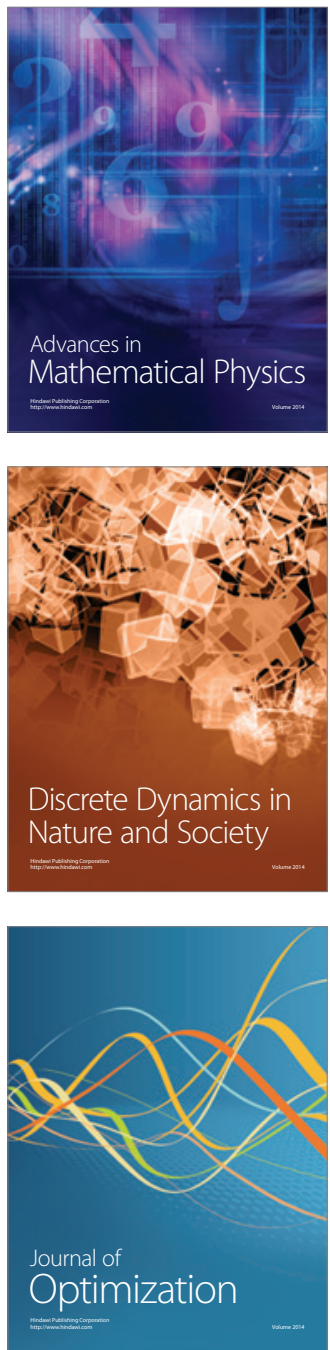